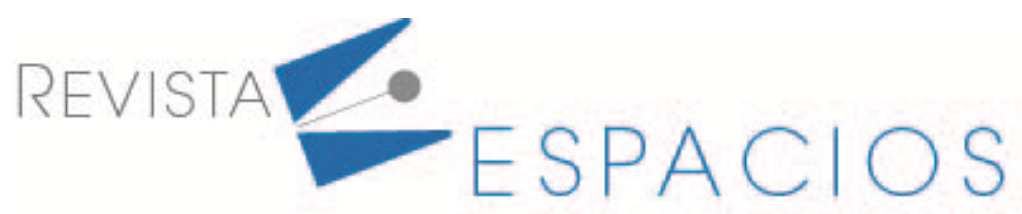

\title{
Impactos de la vivienda en beneficiarios de bajos ingresos en Tunja-Colombia: un estudio focal
}

\author{
Impacts of housing on low-income beneficiaries in Tunja-Colombia: a focal study
}

\author{
MENDOZA VARGAS, Juan M. ${ }^{1}$ \\ BURBANO PANTOJA, Víctor M. ${ }^{2}$ \\ MENDOZA VARGAS, Hugo $\mathrm{H}^{3}$
}

\begin{abstract}
Resumen
Se presenta el estudio del impacto en un grupo de propietarios de vivienda para familias de bajos ingresos en Tunja-Colombia. La metodología asume un enfoque mixto de investigación con diseño de corte transversal y encuesta a 82 cabezas de hogar. Los resultados mostraron la existencia de impactos económicos, psicosociales y ambientales en los hogares beneficiarios. Se concluye que la vivienda adquirida ha afectado a sus usuarios de distinta forma: directa, indirecta, positiva o negativamente.

Palabras clave: impactos, calidad de vida, vivienda de interés social, hogares beneficiados
\end{abstract}

\begin{abstract}
This article reports on various impacts that emerge from the information provided by a group of homeowners for low-income families in Tunja-Colombia. The methodology assumes a mixed research approach with a cross-sectional design and a survey of 82 heads of households. The results showed the existence of economic, psychosocial and environmental impacts in the beneficiary households. It is concluded that the acquired home has affected its users in different ways: directly, indirectly, positively or negatively.
\end{abstract}

Keywords: impacts, quality of life, affordable housing, beneficiary households

\section{Introducción}

Probablemente, la vivienda es la más grande inversión que un gran número de hogares hacen a lo largo de toda su vida (Cuervo y Jaramillo, 2009); algunos hogares con ingresos medios pueden tardar más de 25 años en pagarla, un porcentaje pequeño de hogares con bajos ingresos quizá lo haga en 30 o más años (Fuentes et al., 2020) y el resto posiblemente nunca lo haga y vivan como inquilinos ya que reflejan pocas condiciones para adquirir una vivienda propia (Jaramillo \& Ibáñez, 2002) y prioritariamente piensen en satisfacer sus necesidades básicas. Por la forma como se ha ejecutado la política de vivienda en los últimos diez años en Colombia, paulatinamente la implementación de la Vivienda de Interés Social (VIS) ha quedado en manos de los

\footnotetext{
${ }^{1}$ Administrador de empresas, Magíster en Procesos de dirección empresarial. Facultad de Estudios a Distancia (FESAD). Universidad Pedagógica y Tecnológica de Colombia, calle 48 No. 8-48, Tunja, Colombia. juanmaria.mendoza@uptc.edu.co

${ }^{2}$ Magíster en Estadística, Doctor en Ciencias de la Educación. Escuela de Matemáticas y Estadística. Universidad Pedagógica y Tecnológica de Colombia, calle 48 No. 8-48 Tunja, Colombia. victor.burbano@uptc.edu.co

${ }^{3}$ Licenciado en Ciencias de la Educación-Física y Matemáticas, Magíster en Metalurgia y Ciencia de los materiales. Facultad de Estudios a Distancia (FESAD). Universidad Pedagógica y Tecnológica de Colombia, Tunja, Colombia. hugo.mendoza@uptc.edu.co
} 
intermediarios, en lo referente al manejo financiero y los procesos de construcción (Robles y Robles, 2019), con implicaciones inmediatas sobre los aspectos económicos, sociales y ambientales de los usuarios de la VIS, quienes padecen diversas tragedias durante el procesos adjudicatorio y en el transcurso del pago de la VIS por tratarse de personas de bajos ingresos o de familias vulnerables que ha de lidiar con las inclemencias del capital financiero (Moser \& Dani, 2008).

Además, diversas clases de incumplimiento se han detectado en los contratos celebrados entre el estado y algunas empresas constructoras de VIS, los cuales han generado diferentes percepciones y afectaciones sobre la vida de los beneficiarios de tales viviendas, quienes manifiestan distintos tipos de insatisfacción, unos respecto a los procesos de adjudicación de la VIS y las características físico-espaciales (Mendoza et al., 2020), y otros relacionados con la financiación y habitabilidad de la vivienda adjudicada. En este contexto, la pregunta que orientó el proceso investigativo fue ¿cuáles son los principales impactos que emergen de la información aportada por los beneficiarios de la VIS adjudicada a través de los proyectos EDR y TDP (seudónimos) en la ciudad de Tunja en Colombia? La respuesta no es inmediata y se requiere adoptar un marco teórico que permita dilucidar tales impactos con base en las percepciones de los usuarios en torno a las condiciones físicas de la VIS que habitan, el contexto del barrio y las realidades tanto económicas como psicosociales por las cuales atraviesan actualmente los beneficiaros de la VIS en tales proyectos.

\subsection{Marco teórico}

La constitución colombiana de 1991, en su artículo 51 consagra el derecho a que los ciudadanos puedan acceder a una vivienda digna (Mejía, 2016) y de esa forma, promover el desarrollo armónico de la familia, con la posibilidad de disfrutar de los bienes de capital o de la VIS para quienes provienen de poblaciones vulnerables; en general para la adquisición de la vivienda se requiere del capital financiero del estado y de los usuarios (Moser \& Dani, 2008); sin embargo, las familias de bajos ingresos aspiran a los subsidios otorgados por estado para adquirir su VIS (Herrera, 2015) o a los créditos de bajo costo; con la aspiración de menguar su estado de pobreza e inquilinato cotidiano (Salles y López, 2009).

Para las personas de bajos ingresos, la vivienda se ha constituido en el principal trasmisor de satisfacción e identidad individual y familiar, generándose diversos impactos en correspondencia con las características del hábitat, tamaño, diseño e insumos usados en su construcción y costo final de la misma. Aspectos como el crecimiento macroeconómico del país, el empleo, la distribución del ingreso y el mercado de la VIS también han afectado directamente a las familias más pobres; y de hecho, a la eficiencia en la política de vivienda emanada por el estado; estos factores producen mayor desigualdad social, pobreza, necesidades básicas insatisfechas y problemas psicosociales (Fidel et al., 2018); en consecuencia, es perentorio, reorientar la política subsidiaria neoliberal para la VIS de modo que, permita concertar escenarios más justos e igualitarios para su acceso (Petersen, 2015; Hidalgo et al., 2016), financiación y sostenibilidad ambiental.

Generalmente, los usuarios esperan que su VIS posea características físico-espaciales y ambientales que le aporten condiciones de dignidad, igualdad, equidad y bienestar a sus familias de modo que, puedan integrarse de mejor manera a la sociedad, en contraposición al inconformismo y las reclamaciones desgastantes que se presentan cuando los constructores entregan las VIS parcialmente terminadas, se retrasan en las obras o no cumplen con su entrega (Fuentes et al., 2020; Mendoza et al., 2020); estos factores junto al frecuente déficit en el presupuesto familiar, la baja capacidad de ahorro y la satisfacción parcial de las necesidades básicas del usuario VIS, le generan impactos socioeconómicos y psicosociales desfavorables para los miembros del núcleo familiar (Valera, 2019).

Por otra parte, las VIS se han construido tanto en altura como superficie y como parte de conjuntos residenciales sin consultar las realidades de los usuarios, su organización familiar, la adaptación con el lugar, las facilidades de 
movilidad tanto internas como externas a través de los medios de trasporte, el grado de densificación social y arquitectónico, la comunicación con los vecinos, el acceso a los servicios básicos, centros comerciales y otros que generan esparcimiento como campos deportivos y lugares de diversión (Marengo, et al., 2018); estos aspectos pueden impactar la satisfacción residencial del usuario. Por lo tanto, la planificación del modelo de VIS debe consultar la realidad económica y social de los beneficiarios en correspondencia con la capacidad financiera asignada por el gobierno colombiano, la responsabilidad social de las constructoras (Pérez, 2016), las características físico-espaciales y funcionalidad de la vivienda.

También, Caldera y Valecillos (2003) afirman que por su cultura, costumbres y diversos lugares de procedencia, los usuarios VIS prefieren vivir en viviendas unifamiliares antes que en altura (torres de apartamentos), este aspecto también puede impactar la forma de vivir y trabajar de los beneficiarios. En consecuencia, la planificación de la VIS ha de atender tanto las aspiraciones de movilidad al interior de la vivienda y el bienestar del usuario (Gopalan y Ventakaraman, 2015) como la posibilidad de acceder con mayor facilidad a su lugar de trabajo y los servicios básicos (Rincón y Robledo, 2016). En este sentido, las entidades financieras y el gobierno deben hacer un mayor esfuerzo e intervenir el mercado de la VIS a fin de mejorar la calidad de vida de las familias de bajos ingresos, evitar la proliferación de asentamientos suburbanos (Camelo y Campo, 2016) y favorecer en alguna medida las precarias condiciones socioeconómicas de los usuarios de la VIS.

Por otro lado, la naturaleza psicosocial de la vivienda debe atender conformidades que satisfagan los componentes del saber, pensar y sentir de la persona, entre otros, la apreciación, conciencia y evaluación de la VIS tanto en el contexto interno como externo para que genere condiciones de dignidad (Wapner, 1991); en este sentido, la VIS ha de permitir la movilidad física de quienes la habitan, debe disponer de las unidades facilitadoras del proceso de circulación y comunicación entre usuarios (Peponis y Wineman, 2002), ha de posibilitar la interacción del núcleo familiar en un ambiente agradable para el diálogo, ha de constituirse en un lugar donde se pueda reposar, residir, comunicarse y permanecer sin tensiones externas, proporcionando tranquilidad y paz a quienes la habitan. Estos aspectos son prioritarios y han de tenerse en cuenta desde la política social de vivienda (Pinto de Carvalho y Cornejo, 2018) de modo que la VIS se constituya en el lugar donde se fusione el diseño arquitectónico con el bienestar psicosocial de la familia.

Además, el vecindario donde se ubica la VIS impacta directamente sobre sus características ambientales y los procesos de convivencia entre los usuarios y su entorno natural y social (Florian, 2011). Este aspecto ha de tenerse presente en los diseños arquitectónicos de la VIS de modo que, también permitan conservar el escenario con las facilidades de comunicación con diversas áreas del entorno (Herrera y García, 2014). Asimismo, las características de una VIS digna han de prevalecer en tanto que han sido instituidas por los organismos internacionales defensores de los derechos humanos; tales características se han de ajustar al costo de 135 salarios mínimos vigentes fijados por el gobierno colombiano pero han de proteger al usuario de los factores climáticos adversos, los riesgos de colapso y vectores de propagación de enfermedades (Díaz y Luna, 2011).

En lo que respecta, a la financiación de la vivienda, en Colombia y en los países subdesarrollado las tasas de interés en los productos financieros destinados a la VIS para los hogares vulnerables, son más elevadas que para las familias de los países ricos; esta situación también impacta las finanzas de los hogares con bajos ingresos puesto que, resulta muy difícil que consigan un préstamo por los múltiples requisitos que exige el sector bancario (García y Gómez, 2016), en este caso, los hogares se ven obligados a adquirir créditos en diferentes modalidades con particulares, lo cual precariza su economía familiar y en algunos casos lleva a la pérdida de la VIS adquirida, cuando caen en situaciones de mora tanto en las cuotas como en los intereses (Ruprah, 2010) puesto que el indicador de ahorro está en relación de la capacidad económica de las familias y directamente proporcional a sus ingresos. 
Factores como el encarecimiento de los suelos para construcción de vivienda, bajo capacidad de endeudamiento por parte de las familias pobres y la reiterada negativa de los bancos para financiar VIS, obligan a los usuarios a optar por nuevas formas de solucionar su problema habitacional, entre ellos están: la autoconstrucción atándose a la capacidad de ahorro, la solicitud de subsidios de vivienda y créditos hipotecarios con bancos del estado (Gonzales, 2002). En este contexto, el subsidio para VIS se destina a familias con capacidad de endeudamiento (Zúñiga, 2014), luego las familias en condiciones de máxima vulnerabilidad quedan sin oportunidad de acceder a una VIS por encontrarse en condiciones de subempleo o de ingresos a través del trabajo informal.

Para mitigar estos casos en Colombia, conviene reorientar los procesos de adjudicación de la VIS, apalancar los esfuerzos familiares para pagar las obligaciones hipotecarias, condonar algunas deudas, impulsar la VIS gratuita para casos de familias en condiciones de pobreza extrema y ofrecer VIS en la modalidad de arrendamiento, entre otros (Pareja y Sánchez, 2012). El Estado dentro de sus políticas de vivienda debe vincular los subsidios a las iniciativas particulares de los ahorros de los usuarios, facilitar instrumentos de acceso a los créditos como complemento al capital que la familia haya acumulado para su VIS, minimizar los costos, controlar la especulación en el mercado inmobiliario, y reestructurar el modelo de vivienda estandarizado, de mala calidad y de alto costo (Ramírez, 2002) que ha dejado en la miseria a no pocos hogares en las ciudades.

Con base en el derecho constitucional y los tratados internacionales, es perentorio que el estado revise su política de adjudicación de la VIS para que los más pobres puedan hacer efectivo su derecho de adquirir una VIS (Relinque, et al., 2019), con características físico-espaciales y ambientales que dignifiquen su forma de vida, con espacio físico suficiente, vías de acceso pertinentes, con un diseño arquitectónico atractivo que permita la integración social y el disfrute del espacio público que generen espacios urbanos con mecanismos de comunicación y afinidad entre quienes habitan las VIS (García, 2018). Sin embargo, en Colombia se ha adoptado la estrategia de densificación habitacional en sentido vertical (bloques de apartamentos reducidos) que genera ganancias para los constructores e impactan negativamente a las familias con bajos ingresos, traduciéndose en baja calidad en la distribución interna y en las áreas sociales construidas, bajo una perspectiva tradicional ineficaz (Rubio, 2009).

Tambien, Montaner (2010) afirma que el modelo arquitectónico también debe ajustarse a las características antropológicas del usuario y a las condiciones físicas del lugar de modo que, se conjuguen sus actividades diarias con su desarrollo psicosocial y su entorno medioambiental; de allí la necesidad de que los usuarios participen en las etapas de planeación y construcción de su VIS para que esta sea funcional y acorde a sus necesidades, de ese modo se generará un impacto positivo para la familia del usuario; cuando se desconocen las conductas habituales de los futuros residentes, generalmente se producen impactos psicosociales de incompatibilidad entre familia y espacio físico. Por lo anterior, el diseño de la VIS requiere con urgencia considerar los diversos aspectos antropológicos asociados con la cultura del usuario y el crecimiento demográfico (Brausch y Katunsky, 2015; Grueso y Antón, 2015).

Sarquis (2007) afirmaba que no existía un diseño único en la planificación de la VIS puesto que debía estar focalizado en el progreso económico, social, cultural y ambiental de las familias menos favorecidas; en este sentido resulta razonable la participación ciudadana en la construcción de ciudad (Navarrete, 2017) y en la interacción del usuario con las constructoras a fin de diseñar un mejor hábitat, centrado en los diversas formas de vida de los beneficiarios y las técnicas de construcción dinámicas, modernas y ecológicas que deberían caracterizar al mercado inmobiliario (Fernández, 2015). Así entonces, la VIS debe proyectarse vinculando los principios fundamentales de la arquitectura pero atendiendo a las necesidades de los usuarios y al crecimiento urbano, donde se conjuguen aspectos materiales, económicos, psicológicos, sociales y antropológicos que favorezcan el proyecto de vida familiar (Fiscarelli, 2018) de manera que se generen impactos positivos. 
En esta dirección, el diseño de la VIS debe partir de la consideración de características cualitativas que garanticen su vida útil y al mismo tiempo provean satisfacción a quienes la habitan, entre las características internas están: el tamaño, la configuración estética y la comunicación entre sus espacios físicos: cocina, baños, dormitorios, comedor, sala-comedor (Navarrete, 2017), entre otros; también deben ser analizadas las cualidades asociadas con factores externos como la iluminación, aireación, audición y calefacción que armonizan el ambiente y generan condiciones pertinentes para una buena habitabilidad de la VIS (Matamoros y Gutiérrez 2013), en cambio, cuando las obras son entregadas de manera inconclusa o con fallas por parte de las constructoras, generalmente producen impactos negativos.

Finalmente, el confort habitacional está asociado con características deseables en la VIS que posibiliten el bienestar personal de cada uno de los integrantes de la familia, otorguen al usuario el título de propiedad respectivo, dispongan de servicios domésticos adecuados para su funcionamiento, faciliten la movilidad tanto interna como externa, y sobre todo mejoren la calidad de vida familiar. En contraste, Salles y López (2009) señalan que una vivienda inadecuada y empobrecida priva de oportunidades de desarrollo integral a sus ocupantes, los impacta negativamente tanto en lo económico y psicológico como en los psicosocial y ambiental, prolifera la insatisfacción y se acrecientas los riesgos psicosociales (Fernández, 2016). Este marco de referencia proporcionó elementos conceptuales para elaborar una exploración sobre los impactos de la VIS adjudicada en una muestra de usuarios de dos proyectos en la ciudad de Tunja.

\section{Metodología}

En este apartado se indica el enfoque investigativo, el grupo focal objeto de estudio, los procedimientos desarrollados, instrumentos utilizados para recolectar la información y la forma como se elaboró el análisis de los datos.

Este trabajo tuvo un enfoque mixto de investigación, focalizado tanto en los métodos descriptivos de datos cuantitativos como cualitativos (exégesis textual) para el análisis de información; en concordancia con Hernández et al. (2003), en los métodos cuantitativos, la información se recoge por medio de variables con el fin de explorar aspectos puntuales acerca de los individuos de un grupo específico (Burbano y Valdivieso, 2016), y en los métodos cualitativos, se elabora un análisis de tipo interpretativo sobre las frases expresadas por los participantes (Burbano, 2017); también se efectuaron procesos de confrontación de informaciones provenientes de una encuesta y el diálogo directo del investigador con los usuarios (Campos, 2009) para enriquecer la exploración de los hallazgos.

El grupo focal objeto de estudio se constituyó por 82 cabezas de familia, propietarios de la VIS adjudicada por el Estado colombiano, seleccionados por conveniencia, quienes accedieron de forma verbal a dar un consentimiento informado y contestaron de forma individual y por voluntad propia a los interrogantes formulados por medio de una encuesta y el diálogo investigador-miembros del grupo focal. La encuesta se conformó por 13 preguntas. Tal encuesta fue sometida al criterio de jueces expertos, quienes elaboraron algunas recomendaciones tendientes a mejorar el contenido de las preguntas; dichas recomendaciones fueron incorporadas a la encuesta que se aplicó posteriormente a los participantes.

En cuanto a los procedimientos desarrollados en este trabajo, en primer lugar, se elabora un marco teórico que posibilitaría entender el problema planteado y en segundo lugar discutir los hallazgos en torno a los impactos generados por la VIS. El procesado de la información se hizo mediante el Excel y con el software R. Se estructuró un análisis exploratorio-descriptivo a partir de los resultados, el cual se focalizó en generar gráficas e interpretar porcentajes asociados con la información aportada por los encuestados y el diálogo investigador-participantes en este estudio (Mendoza et al., 2020). La exégesis textual de la información recolectada se complementó con algunos aspectos conceptuales aportados por los autores referenciados en el marco teórico. 
Finalmente, emergieron los aspectos relacionados con el impacto percibido por los usuarios con respecto a la VIS adquirida en correspondencia con los entornos tanto interno como externo de la misma, las características físico-ambientales y socioeconómicas y de ubicación de los conjuntos residenciales de donde se tomó la muestra. La investigación también recoge aspectos asociados con el tipo de ingreso, las necesidades básicas de los usuarios y la percepción de los encuestados en cuanto a la localización de la vivienda, sitios adyacentes a las áreas comunes y accesos a la urbanización objeto de estudio.

\section{Resultados}

A continuación se presentan los resultados de este trabajo investigativo focalizados en las respuestas aportadas por los encuestados y asociados con los impactos que la VIS adjudicada ha generado sobre los usuarios.

En este estudio, se encontró que (ver Figura 1), el jefe del núcleo familiar sustenta las necesidades básicas de su familia y la responsabilidad económica asociada con la VIS adquirida con ingresos provenientes de contratos fijos (29\%) o temporales (29\%), trabajo por cuenta propia (24\%), labores del hogar (13\%) y en algunos casos con trabajo que se realiza de forma esporádica (4\%); en este contexto, se evidencia que, los usuarios de VIS tienen que hacer grandes esfuerzos para cubrir sus necesidades habitacionales pero menguando el ingreso destinado a solventar sus necesidades básicas.

Figura 1

Origen de los ingresos del jefe del núcleo familiar para el sustento de la familia

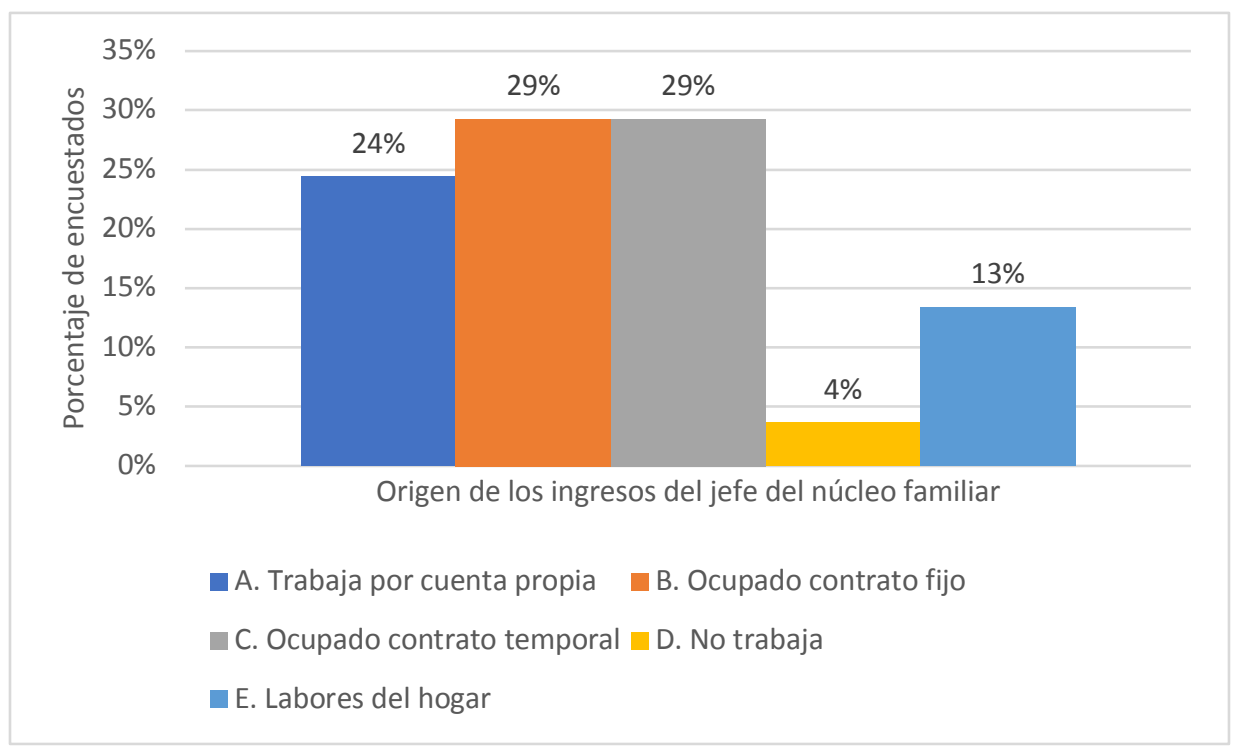

Fuente: Los autores

Por otra parte solamente un $24 \%$ de los encuestados gana más de un salario mínimo, el $48 \%$ percibe un salario mínimo y el resto (15\%) se las tiene que arreglar con menos de un salario mínimo; esto indica que, un alto porcentaje de los beneficiarios pasa dificultades para alimentarse, educarse o adquirir una VIS como una de las necesidades básicas parcialmente satisfecha (ver Figura 2). 
Figura 2

Ingresos mensuales del jefe del hogar

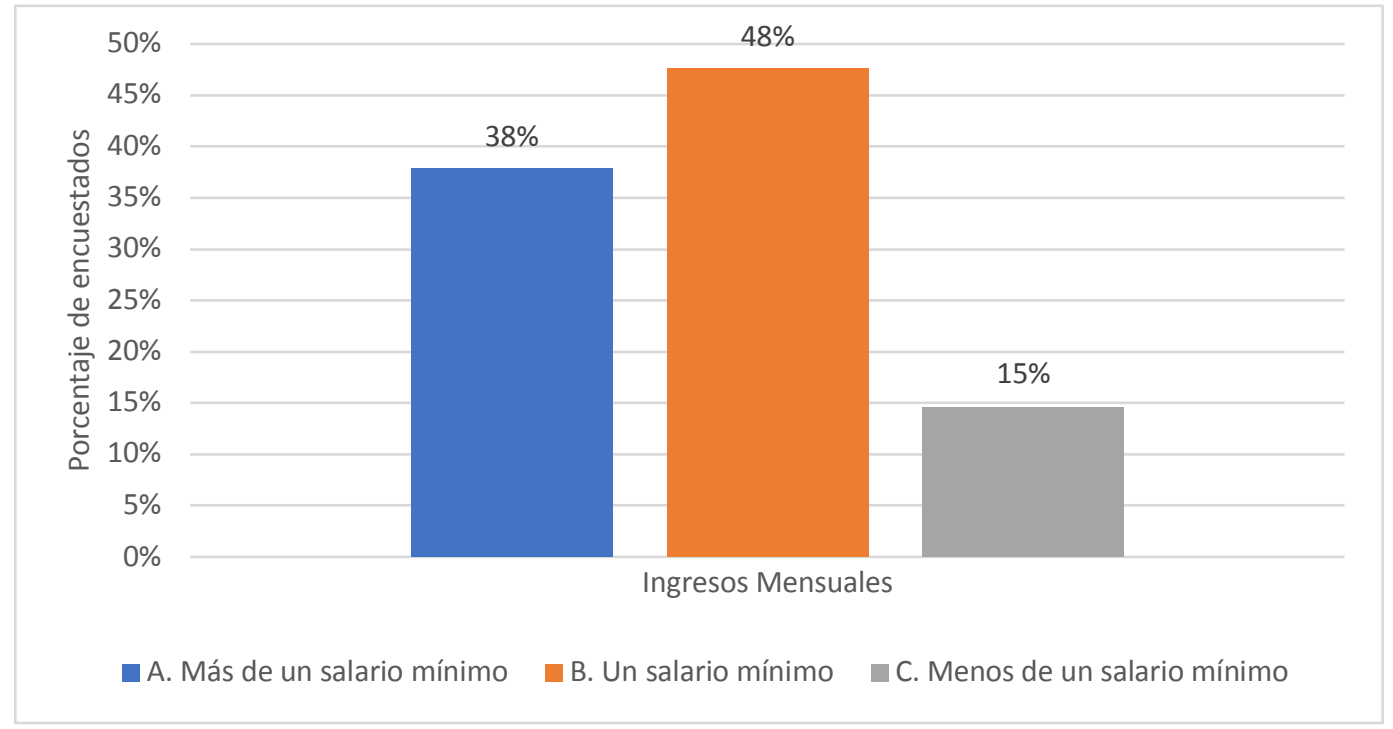

Fuente: Los autores

En referencia al origen de los recursos financieros para la adquisición de la VIS, el 30\% de los usuarios indica que fueron beneficiarios de las ayudas del gobierno a manera de subsidios, el $29 \%$ utilizó sus propios recursos económicos, el $28 \%$ usó créditos blandos y el $4 \%$ vendió su vivienda anterior para adquirir su VIS (ver Figura 3 ).

Figura 3

Origen de los recursos financieros para la adquisición de la vivienda

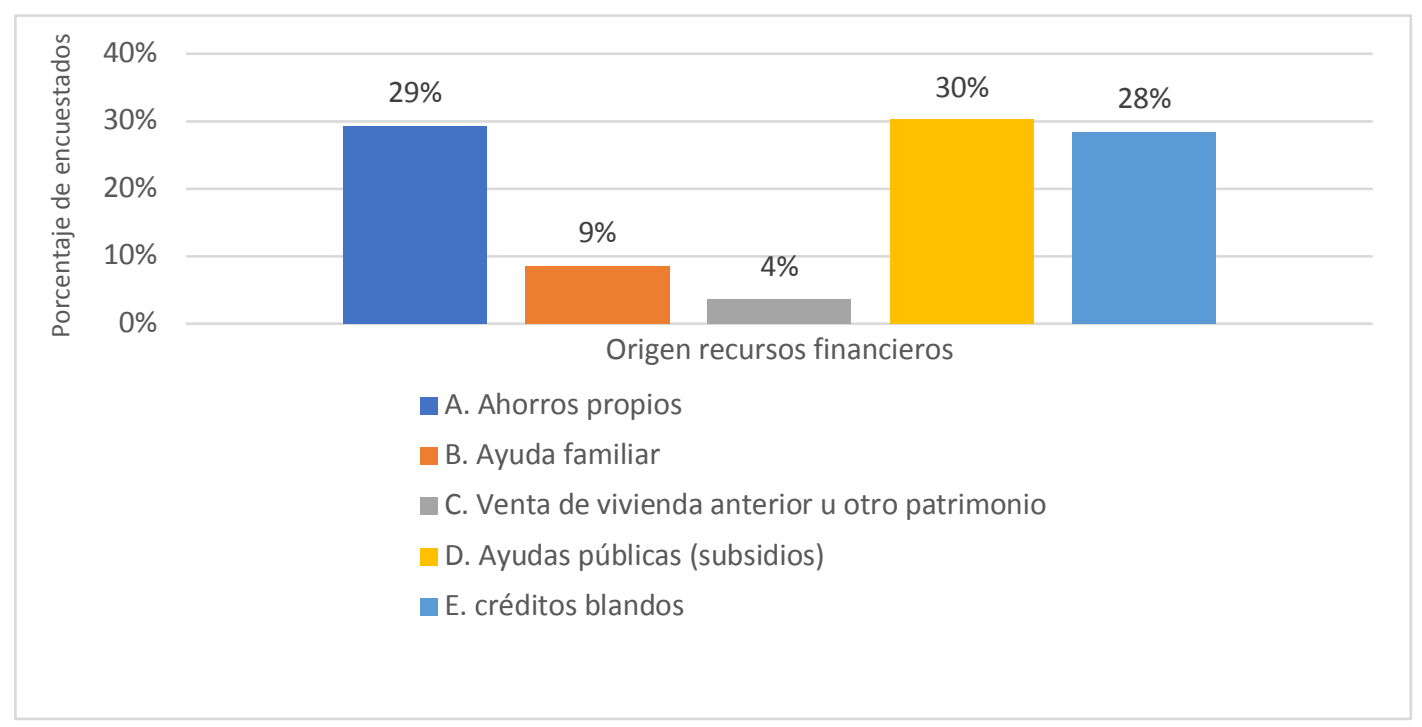

Fuente: Los autores

Un 66\% de los encuestados menciona que, si se ha tomado alguna iniciativa para mejorar la calidad y la eficiencia de las viviendas construidas, ellos mismos han tenido que realizar las reparaciones requeridas para evitar el deterioro de la VIS y el 34\% indica lo contrario (ver Figura 4), lo anterior, debido a que en varios casos, la constructora entregó la VIS sin los terminados acordados previamente. 


\section{Figura 4}

Iniciativas para mejorar la calidad y la eficiencia en la reparación de las viviendas construidas, por parte de los propietarios

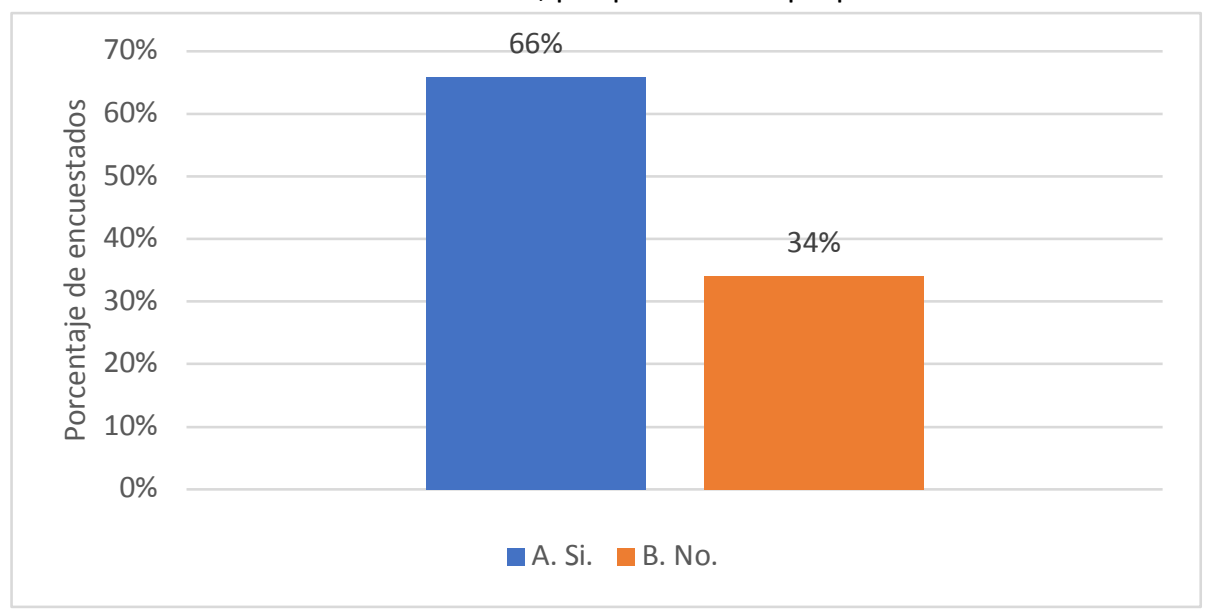

Fuente: Los autores

Adicionalmente, los encuestados manifiestan que su VIS se encuentra ubicada en un medio ambiente con presencia de algunos problemas de alto impacto ambiental tales como proliferación de insectos y roedores (55\%), estar cerca de los botaderos de basuras (33\%) y la falta de alcantarillado (5\%), aunque con menor impacto también está presente la contaminación del aire (ver Figura 5).

Figura 5

Principales problemas del medio ambiente detectados en el ámbito local de la vivienda

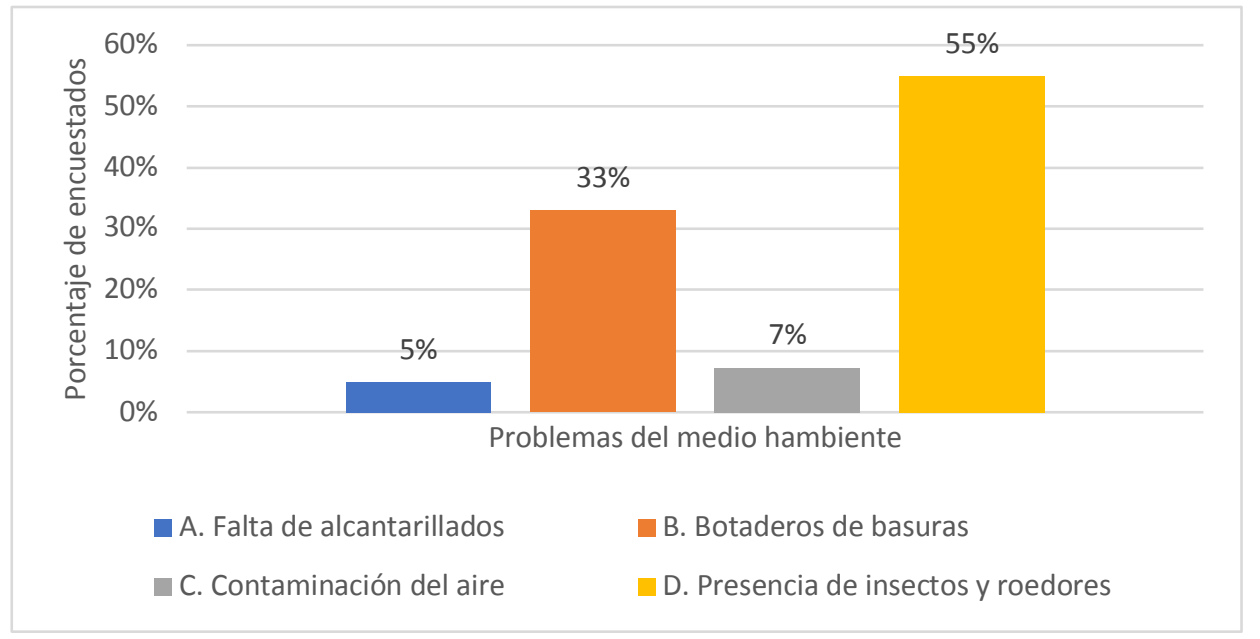

Fuente: Los autores

Por otro lado, a nivel del sector público para los beneficiarios de estos proyectos VIS se han definido algunas estrategias focalizadas en la educación para la convivencia tales como: reforzamiento de la acción comunitaria (35\%), desarrollo de entornos saludables (26\%), refuerzo del entorno familiar (24\%) y desarrollo de habilidades personales (ver Figura 6). 


\section{Figura 6}

Definición de programas púbicos y estrategias

de educación para la convivencia en el barrio

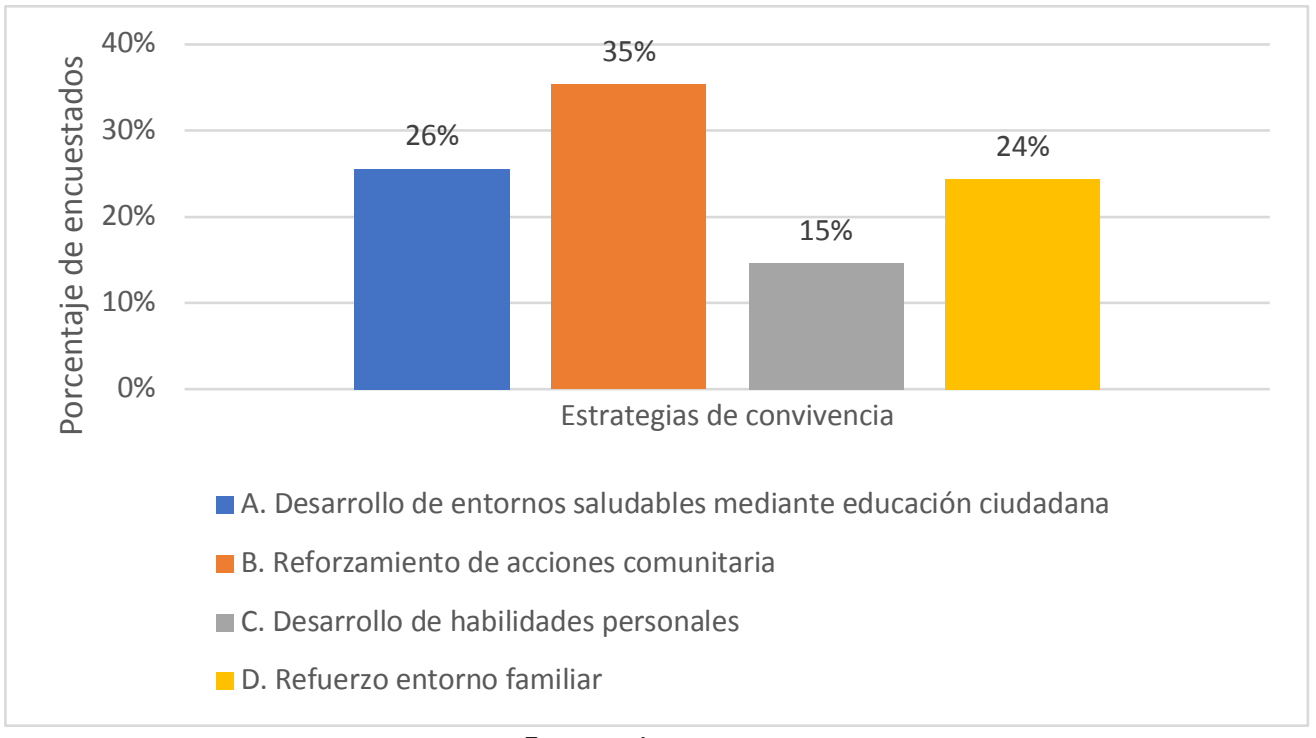

Fuente: Los autores

El 57\% de los encuestados indica que si se han aplicado las normas existentes para exigir el cumplimiento de la entrega de las viviendas pendientes, el $27 \%$ considera que se deben construir la totalidad de las viviendas y el $16 \%$ se acoge a tales normas para exigir la devolución de los aportes que ellos habían hecho (ver Figura 7).

Figura 7

Normas o instrumentos creados para cumplir con la calidad y las entregas pendientes de viviendas a nivel local y nacional

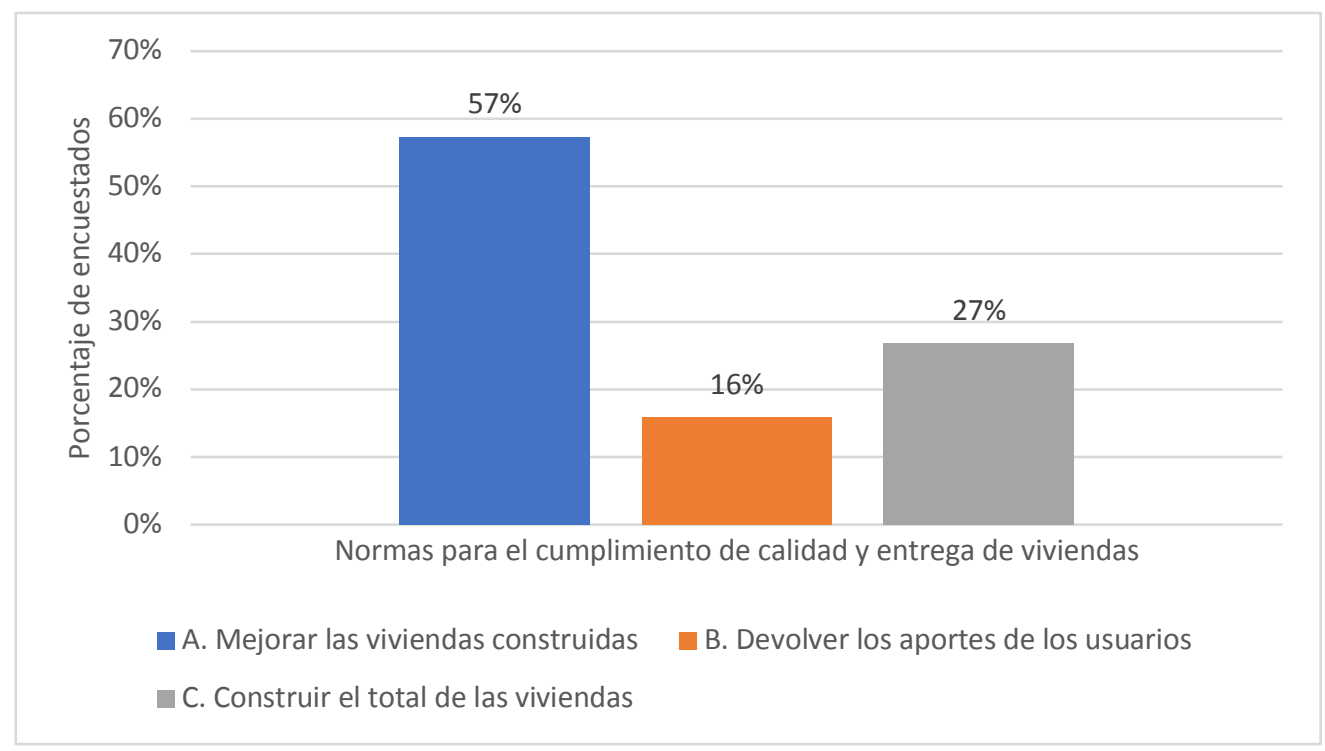

Fuente: Los autores

El 55\% de los entrevistados manifiesta haber existido un bajo nivel de participación social y toma de decisiones en la planificación, control y seguimiento de los proyectos de vivienda, el $43 \%$ piensa que fue nulo y solamente un $2 \%$ considera que fue alto (ver Figura 8 ). 
Figura 8

Nivel o grado de participación social en el análisis y toma de decisiones

en la planificación y control del seguimiento del proyecto de vivienda

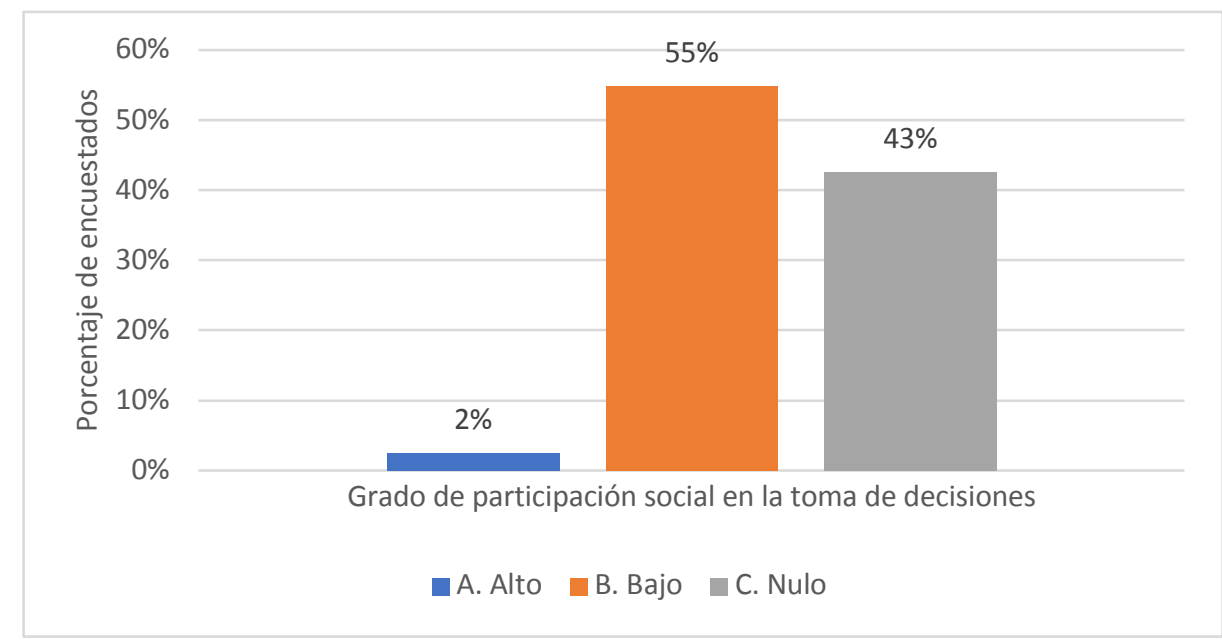

Fuente: Los autores

En lo referente a la parte interna de la VIS, el $36 \%$ de los encuestados indicaron que harían cambios en la cocina, el $26 \%$ modificaría el baño, el $21 \%$ modificaría las puertas y ventanas y el $17 \%$ haría arreglos de las instalaciones de agua y electricidad. Estos hallazgos evidencian las erogaciones que debieron efectuar los usuarios con el fin de optimizar los aspectos internos de su VIS, de modo que se adecuara a su forma particular de vida matizada por su cultura; estos gastos impactan fuertemente a la economía del hogar, su bienestar dentro de la VIS y la satisfacción personal de los miembros de la familia (ver Figura 9).

Figura 9

Tipo de rehabilitación o cambios que realizaría al interior de su vivienda

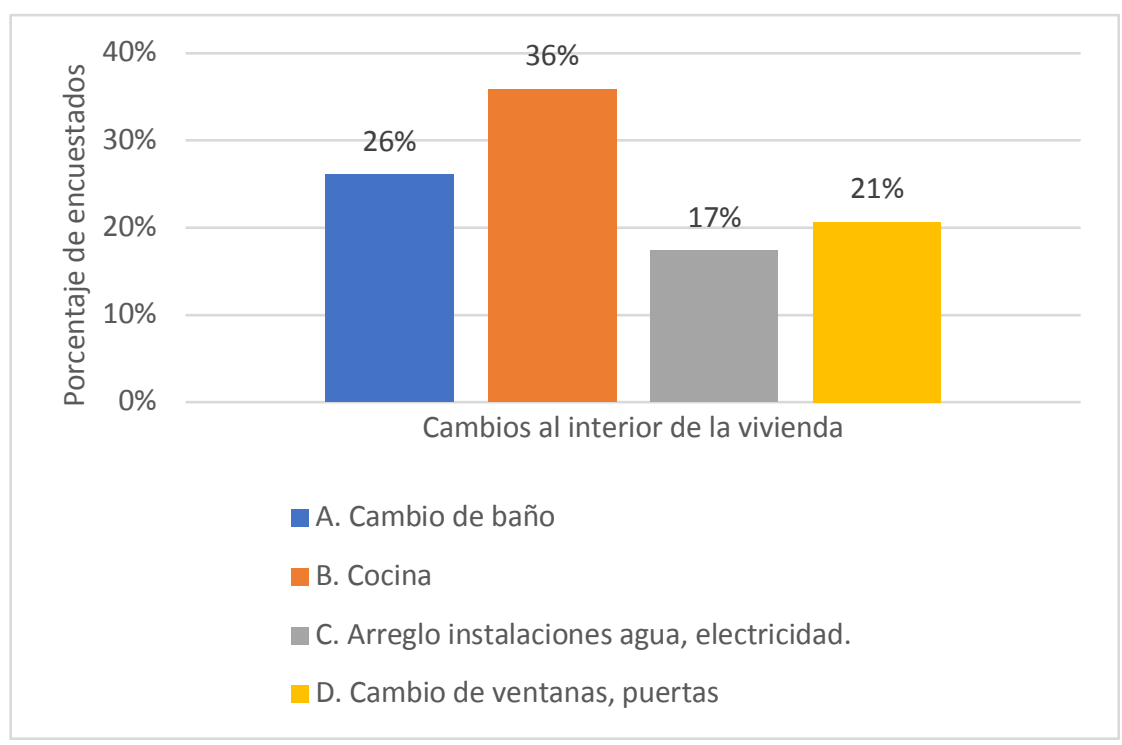

Fuente: Los autores

En cuanto a la parte externa de la vivienda que los beneficiarios de la VIS deseaban mejorar, el $42 \%$ de los encuestados indicaron que, mejorarían las escaleras, el 35\% dijo que la fachada, el $15 \%$ el tejado y el $8 \%$ el ascensor. Estos hallazgos evidencian la preocupación de los usuarios por mejorar aspectos de primera necesidad, los cuales prevalecen sobre los aspectos suntuarios; estos aspectos impactan tanto la economía familiar como los referidos al entorno ambiental y social del barrio (ver Figura 10). 
Figura 10

Parte externa de la vivienda que el usuario le mejoraría

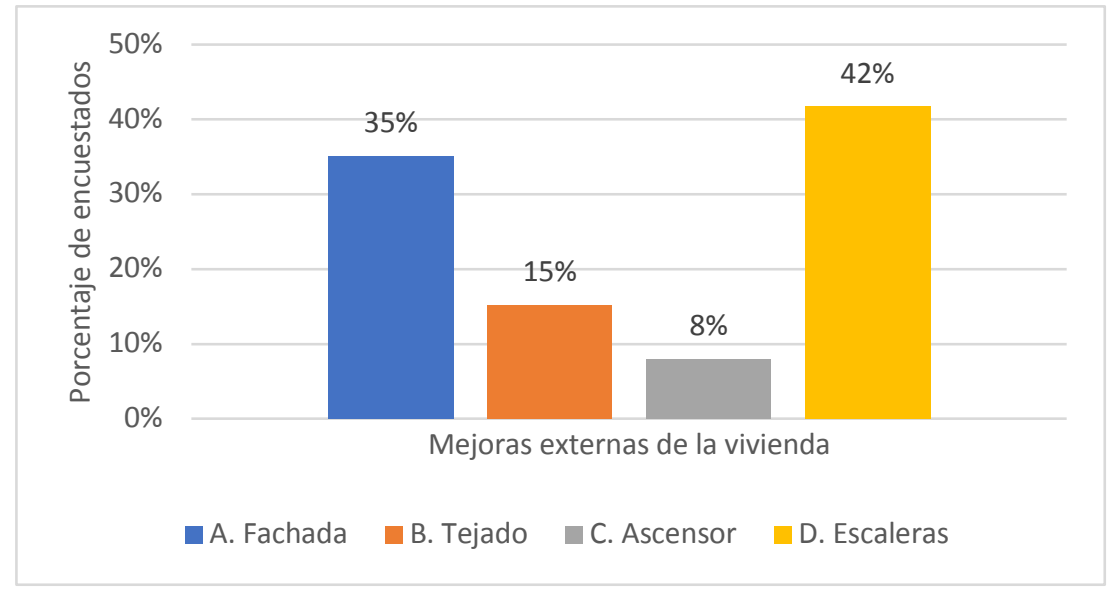

Fuente: Los autores

Sobre los grupos sociales han tenido prioridad en la asignación de los cupos de vivienda, un $44 \%$ de los encuestados indica que el grupo correspondiente a las mujeres cabeza de familia, un $39 \%$ a los trabajadores, un $17 \%$ a los migrantes y el $1 \%$ a los habitantes de calle; en estas circunstancias, la adjudicación de la VIS ha impactado a los diversos grupos sociales que viven en el vecindario (ver Figura 11).

\section{Figura 11}

Grupos sociales que han tenido prioridad en la asignación de los cupos de vivienda

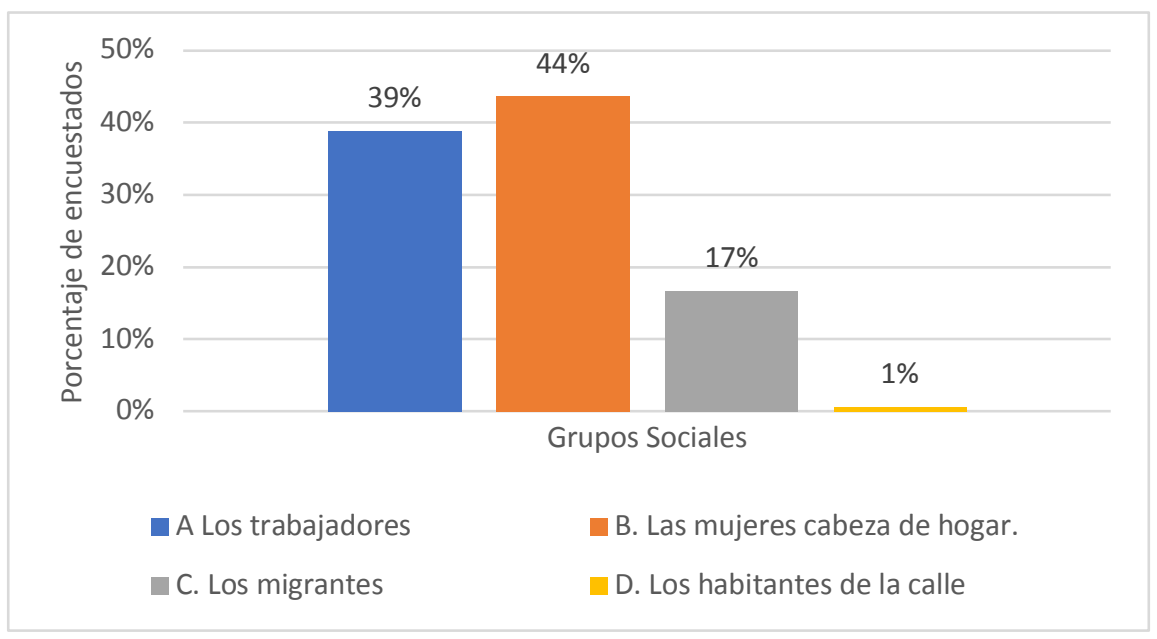

Fuente: Los autores

El 55\% de los encuestados menciona que están satisfechos con las características físico-espaciales de su vivienda actual y un $45 \%$ opinan lo contrario; estos hallazgos evidencian que hay una tendencia a que su VIS los haya impactado de manera positiva desde el punto de vista físico y espacial, lo cual puede generar un ambiente agradable para habitar su vivienda (ver Figura 12). 
Figura 12

¿Usted está satisfecho con las características

físico-espaciales de su vivienda actual?

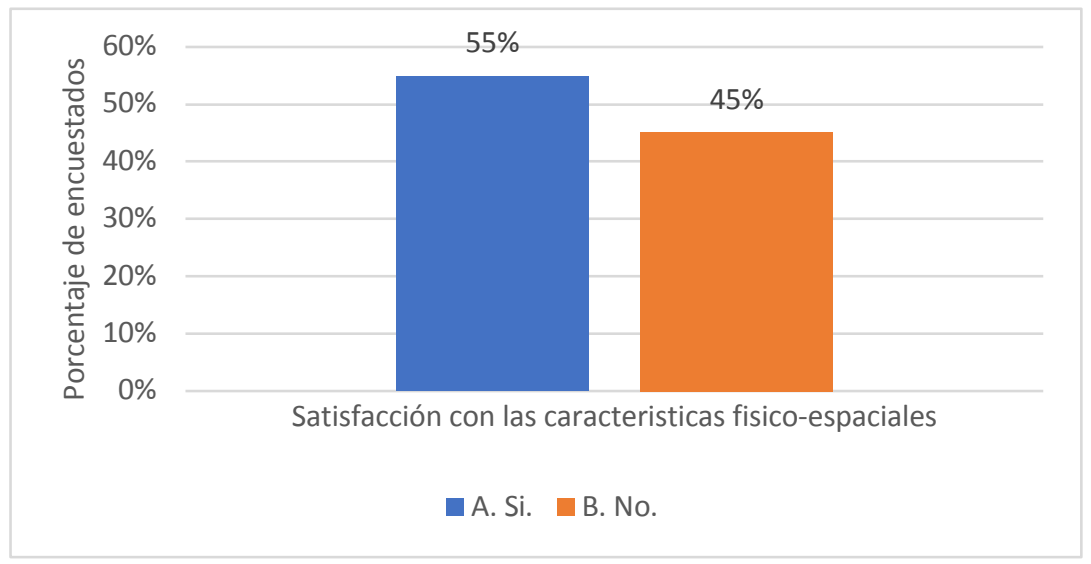

Fuente: Los autores

Finalmente, el $57 \%$ de los beneficiarios de la VIS menciona que, no están satisfechos con las características socioambientales de su vivienda actual y el $43 \%$ percibe lo contrario. Estos aspectos impactan de manera desfavorable sobre el bienestar emocional de las familias que habitan la VIS de los proyectos objeto de estudio (ver Figura 13).

Figura 13

¿Usted está satisfecho con las características

socio-ambientales de su vivienda actual?

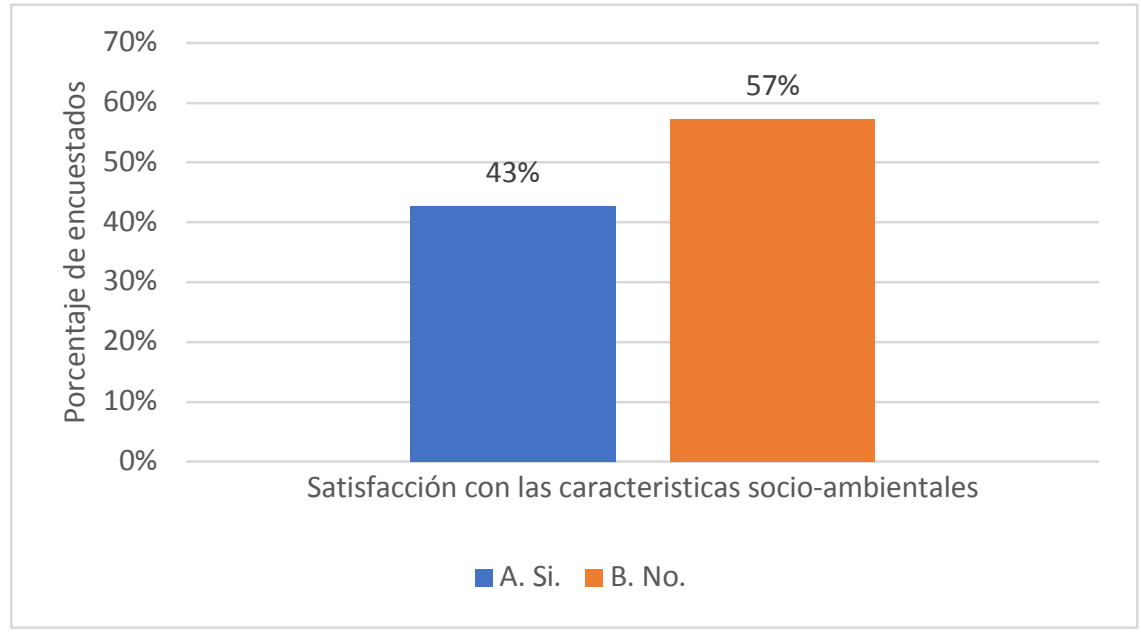

Fuente: Los autores

\section{Discusión}

En este trabajo se exploraron los diversos impactos percibidos por los usuarios respecto a la VIS que les fue adjudicada. Los beneficiarios reconocen el esfuerzo tanto económico como político que ha hecho el Estado colombiano para adjudicarles una VIS y mejorarles su calidad de vida (Serrano et al, 2019); sin embargo, un buen número de ellos sienten que su vivienda actual aún requiere mejoras de distintos tipos para que se constituya en una vivienda que les permita vivir más dignamente (Mejía, 2016) y les ayude a superar el estado de pobreza en que se encuentran. En este contexto, cerca del $63 \%$ de los beneficiarios tiene un ingreso menor o igual a un salario mínimo, con el cual debe satisfacer sus necesidades básicas, cubrir algunas cuotas de su VIS o realizar algunas adecuaciones físico-espaciales porque la VIS adquirida debió entregarse en mejores condiciones por parte de los constructores. 
Estos aspectos, los han impactado tanto en lo económico como en lo ambiental y psicosocial; en algunos casos de manera favorable pero en otros de forma perjudicial en tanto que han menguado su economía familiar y su bienestar social; tales impactos son semejantes a los vividos en proyectos similares ejecutados en otros lugares del territorio nacional (Ubaque et al., 2014); en estas circunstancias, los organismos del Estado y las empresas constructoras responsables de la adjudicación VIS cumplieron de forma parcial sus compromisos, perjudicando en algunos casos a los usuarios, por ejemplo, algunas viviendas requirieron arreglos en la cocina y en los baños; en otras, corregir problemas de humedad y medio ambiente, solo por mencionar algunos. En este sentido, conviene que la normativa de la VIS y la regulación correspondiente se apliquen de forma pertinente y tengan efectos satisfactorios para los usuarios, tutelen los acuerdos pactados entre contratistas, organismos del Estado y usuarios a fin de que estos últimos no se sientan defraudados, generándose impactos psicosociales (Vergara, 2016), por ejemplo, la evidencia empírica mostró que el 16\% de los usuarios exigió la devolución de sus aportes porque no se les entregaron sus viviendas, lo cual no favorece a la resiliencia habitacional ni residencial de las familias de bajos ingresos (Vargas, 2018).

Por otra parte, solamente el $30 \%$ de los encuestados manifiestan haber sido beneficiarios de los subsidios entregados por el estado colombiano, los demás tuvieron que hacer esfuerzos de tipo económico focalizados en el ahorro o la ayuda familiar, los créditos blandos otorgados por el sector financiero, la venta de su vivienda anterior o su patrimonio consolidado por varios años (Gonzales, 2002). Este porcentaje resulta bajo pero ha impactado de manera positiva en el aspecto económico de quienes recibieron los subsidios; en cambio, para aquellos que debieron acudir a los crédito bancarios le ha significado un mayor sacrificio por cuanto mes a mes, deben pagar sus deudas y los intereses con una mengua significativa en la satisfacción de sus necesidades básicas y acrecentamiento de la pobreza (Herrera, 2015).

Por los costos de la construcción de la VIS (135 salarios mínimos) su ubicación no fue la mejor y en su entorno se observa la presencia de acumulación de basuras, falta de alcantarillado para viviendas vecinas, presencia de insectos y roedores que impactan de forma negativa las condiciones medioambientales de las viviendas; por lo tanto ha sido necesaria la participación ciudadana, para que entre todos se pudieran subsanar estos problemas de tipo externo pero que mengua la satisfacción familiar de habitar este sitio (Mendoza, et al., 2020). En este contexto, hacer jornadas para mejorar la apariencia externa de las viviendas y generar un ambiente exterior con menos amenazas para la salud física se ha constituido en una tarea titánica. Por otro lado, también ha sido necesario buscar alternativas para mejorar la apariencia interna de la urbanización y la acción de los propietarios ha propiciado centrar su atención para mejorar las zonas comunes, escaleras y vías de acceso. Estas tareas han significado mejorar la habitabilidad de su vivienda (cocina, baños, instalaciones de agua y electricidad, entre otros) pero con sacrificios tanto económicos como sociales, en tanto que los recursos destinados a recreación y bienestar social, los han tenido que gastar en acondicionar sus viviendas para vivir de una manera más digna (Herrera y García, 2014).

\section{Conclusiones}

Los hallazgos evidencian que el estado actual de las VIS adjudicadas ha generado un alto impacto, tanto directo como indirecto, a los usuarios tanto en sus aspectos económicos y físicos de la VIS como sociales y medioambientales. Las condiciones de alojamiento que ofrece la vivienda a partir de la calidad de los materiales y los procesos constructivos no son los mejores, esto ha implicado tener que conseguir recursos adicionales para mejorar las condiciones de las VIS entregadas, a costas de una mengua de la satisfacción de sus necesidades básicas, situación que en algunos casos ha llevado a precarizar su situación económica. Las condiciones psicosociales y de armonía con su entorno no son óptimas y han generado algunos impactos negativos que no compensan el esfuerzo diario que han debido efectuar los usuarios de la VIS para sufragar gastos adicionales tendientes a mejorar las condiciones físico-espaciales y socio-ambientales de sus viviendas. 


\section{Referencias bibliográficas}

Brausch, C., y Katunsky, D. (2015). Changing social structure in Europe calls for tailor-made, barrier-free living and new interior design. Journal of civil engineering, 10, 75-84.

Burbano V., \& Valdivieso M. (2016). Inferencia Estadística Básica, apoyo al estudio independiente. Tunja, Colombia: Editorial UPTC.

Burbano, V. (2017). Un acercamiento a la didáctica de la probabilidad desde el PKC. Tunja: Editorial UPTC.

Caldera, C. C., \& Valecillos, T. P. (2003). El espacio barrio y su espacio comunitario, un método para la estructuración de lo urbano. Revista invi, 18(48), 78-92.

Camelo, M., y Campo, J. (2016). Análisis de la política de vivienda en Bogotá: un enfoque desde la oferta y la demanda. Revista Finanzas y Política Económica, 8(1), 105-122.

Campos, A. (2009). Métodos Mixtos de Investigación. Bogotá, Colombia: Magisterio

Cuervo, N., \& Jaramillo, S. (2009). Dos décadas de política de vivienda en Bogotá apostando por el mercado (No. 006651). Universidad de los Andes-CEDE. Colombia. Recuperado de: http://ideas.repec.org/p/col/000089/006651.html

Díaz, C., y Luna, R. (2011). Calidad en la vivienda de interés social. Guías de Asistencia Técnica para Vivienda de Interés Social, No. 1. Ministerio de Ambiente, Vivienda y Desarrollo Territorial. Bogotá, D.C. Colombia: Nuevas Ediciones S. A

Fernández, P. (2015). Hacia una vivienda abierta concebida como si el habitante importara. Buenos Aires, Argentina: Diseño

Fernández, G. (2016). El acceso a la vivienda social de las personas sin hogar. Estudio de casos: Alemania, España, Finlandia y Reino Unido. Universitat Autònoma de Barcelona. España

Fidel, C., Di Tomaso, R., \& Farias, C. (2018). Política de hábitat y ensambles urbanos:

¿hacia un modelo extractivista urbano en la Argentina actual? Vivienda y Ciudad, 5, 43-66.

Fiscarelli, D. (2018). Aspectos cualitativos de la vivienda de interés social. ARQUISUR Revista, 8(13), 32-43. https://doi.org/10.14409/ar.v8i13.6817

Florian, A. (2011). Mejoramiento de barrios y viviendas desde la perspectiva del derecho a la vivienda: Oportunidades para una política pública. Ponencia presentada en el Seminario Internacional "Derecho a la Vivienda y Políticas de Hábitat a escala Metropolitana". Medellín, Colombia. Agosto 25 y 26 de 2011

Fuentes, H. J., Contreras, L. E., \& Rodríguez, J. I. (2020). Un análisis del mercado de vivienda en Colombia 19982020. Revista Espacios, 41(31), 267-278.

García, S. (2018). Ciudad, espacio público y hábitat intergeneracional. Estudios del hábitat, 16(1), 1-12.

García, E. A., \& Gómez, A. F. (2016). Mercado de crédito informal en Colombia: una aproximación empírica. Ensayos de economía, 26(49), 137-156.

Gonzales, G. (2002), "El crédito hipotecario y el acceso a la vivienda para los hogares de menores ingresos en América Latina", Serie-CEPAL N¹22, (LC/L.1779-P), Santiago, Chile. (1999), "Alternativas de política para fortalecer el ahorro de los hogares de menores ingresos: el caso del Perú", Serie-CEPAL N85 (LC/L.1245P), Santiago de Chile. 
Gopalan, K., y Venkataraman, M. (2015). Vivienda asequible: política y práctica en India. IIMB Management Review, 27(2), 129-140.

Grueso, M., y Antón, M. (2015). Valores culturales en redes interorganizacionales: Una propuesta desde el Modelo de Shalom Schwartz (1992). Revista Investigación \& Desarrollo, 23(2), 369-390

Hernández, S, Fernández, C., y Baptista, L. (2003). Metodología de la Investigación. México: McGraw-Hill.

Herrera, V. E., \& García, R. C. (2014). Espacios habitacionales, dinamicas sociales y calidad de vida/Social housing, social dynamics and quality of life. RIDE Revista Iberoamericana para la Investigación y el Desarrollo Educativo, 5(9), 128-144.

Herrera, H. (2015). “Notas preliminares para una lectura no-dogmática del principio de subsidiariedad”, en S. Ortúzar y P. Ortúzar (eds.), Subsidiariedad. Más allá del Estado y del mercado, Santiago de Chile: Instituto de Estudios de la Sociedad, pp. 97-112

Hidalgo, R. A., Paulsen, A. G., \& Santana, L. D. (2016). El neoliberalismo subsidiario y la búsqueda de justicia e igualdad en el acceso a la vivienda social: el caso de Santiago de Chile (1970-2015). Andamios, 13(32), 5781.

Jaramillo, S., \& Ibáñez, M. (2002). Elementos para orientar una política estatal sobre alquiler de vivienda urbana en Colombia. Bogotá, Colombia, Centro de Estudios sobre Desarrollo Económico-CEDE, Universidad de los Andes.

Marengo, M. C., Monayar, V., \& Sícoli, M. (2018). Una mirada cualitativa a los modelos de densificación residencial. El caso de la vivienda colectiva en Córdoba-Argentina. Cadernos Zygmunt Bauman, 8(18), 6983.

Matamoros, M., y Gutiérrez, R. (2013). El diseño de interiores en la formación del arquitecto. Experiencias en el Plan de Estudios. Arquitectura y Urbanismo, 34(1), 117-127.

Mejía, M. (2016). La vivienda digna y la vivienda adecuada. Estado del debate. Cuadernos De Vivienda y Urbanismo, 9(18), 292-307

Mendoza, J. M., Burbano, V. M., \& Mendoza, H. H. (2020). Los procesos administrativos en la adjudicación de vivienda de interés social en la ciudad de Tunja (Colombia): una mirada desde los contratistas. Revista Espacios, 41(24), 250-265.

Montaner, Z. (2010). Reflexiones para proyectar viviendas del siglo XXI. Dearq, 6, 82-

99. https://doi.org/10.18389/dearq6.2010.09

Moser, C., \& Dani, A. A. (Eds.). (2008). Assets, livelihoods, and social policy. The World Bank.

Navarrete, M. (2017). Desarrollo urbano sustentable: el gran desafío para América Latina y los preparativos para Hábitat III. Revista Luna Azul, 45(1), 123-149

Pareja E, y Sánchez, M. (2012). La política de vivienda en España: lecciones aprendidas y retos de futuro. Revista Galega de Economía, 21(2), 1-32.

Peponis, J., y Wineman, J. (2002). Spatial structure of environment and behavior. En: Bechtel \& Churchman Eds.). Handbook of Environmental Psychology. New York, U.S.A.: John Wiley \&Sons, Inc.

Pérez, A. L. (2016). El diseño de la vivienda de interés social. La satisfacción de las necesidades y expectativas del usuario. Revista de Arquitectura, 18, 67-75. 
Petersen, M. (2015). "Subsidiariedad, neoliberalismo y el régimen de lo público", en S. Ortúzar y P. Ortúzar (eds.), Subsidiariedad. Más allá del Estado y del mercado, Santiago de Chile: Instituto de Estudios de la Sociedad, pp. 99-167.

Pinto de Carvalho, L., y Cornejo, M. (2018). Por una aproximación crítica al apego al lugar: una revisión en contextos de vulneración del derecho a una vivienda adecuada. Athenea Digital, 18(3), 1-39. https://doi.org/10.5565/rev/athenea.2004

Ramírez, R. (2002). Evaluación social de políticas y programas de vivienda: un análisis de la contribución de la vivienda a la reducción de la pobreza urbana. Revista ini, 17(45), 9-57.

Relinque, F., Vázquez, O., y Fernández, M. (2019). Origen y Evolución de las políticas sociales de vivienda en Europa Cuestión Urbana, 3(5), 63-79.

Rincón, M. C., \& Robledo, J. C. (2016). Análisis de la política de vivienda en Bogotá: un enfoque desde la oferta y la demanda. Revista Finanzas y Política Económica, 8(1), 105-122.

Robles, J. G., \& Robles, J. C. (2019). Los créditos de vivienda de interés social prioritario, las cuotas de amortización y el IVA a los productos de la canasta familiar: un estudio en Colombia en el periodo 2014 2018. Revista Gestión y Desarrollo Libre, 4(7), 1-14.

Rubio, L. (2009). La superación del concepto de "tipo" en la arquitectura contemporánea. Cuaderno de notas, 12, 1-8.

Ruprah, I. (2010). Do social housing programs increase poverty?: An empirical analysis of shelter induced poverty in Latin America. OVE Working Papers 0510. Washington: Inter-American Development BankOffice of Evaluation and Oversight (OVE).

Salles, V., López, M. (2009). Retos para la integración social de los pobres en América Latina. Buenos Aires, Argentina: CLASCO.

Sarquis, J. (2007). Itinerarios del proyecto. La Investigación Proyectual como forma de conocimiento en Arquitectura. Buenos Aires: Nobuko

Serrano, A. M., Tiuzo, S. C., \& Martínez, M. S. (2019). Estrategias Empresariales para una Población Vulnerable en Colombia. Información tecnológica, 30(6), 147-156.

Ubaque, C.A., Trujillo, C.A., Bohórquez, M.L. (2014). Instrumentos de gestión de suelo para vivienda de interés social en Colombia: Análisis de Caso. DYNA: Revista de la Facultad de Minas. Universidad Nacional de Colombia, Sede Medellín, 81(184), 217-224.

Valera, S. (2019). Elementos básicos de psicología ambiental. Barcelona, Univertat de Barcelona.

Vargas, L. (2018). Reflexiones para la construcción de resiliencia territorial, a través del diseño de vivienda social en el posconflicto. In International Conference Arquitectonics Network: Mind, Land and Society, Barcelona, 29-31. Final papers. GIRAS. Universitat Politécnica de Catalunya.

Vergara, A. (2016). Tribunal de contratación pública: bases institucionales, organización, competencia y procedimiento. Revista de Derecho (PUCV), 46(1), 347-378.

Wapner, S. (1991). A holistic, developmental, systems-oriented environmental psychology: Some beginnings. En: D. Stokols and I. Altman. Handbook of Environmental Psychology, 2, 1433-1474. Malabar, Florida: Krieger publishing company

Zúñiga, L. M. F. (2014). Vivienda de interés social: el resultado del presupuesto equivocado. Derecho Público, (32), 23-19. Attribución-NoCommercial 4.0 International

(cc) EY-NC 